\title{
Towards sustainability: artificial intelligent based approach for soil stabilization using various pozzolans
}

\author{
M. S. Ouf \\ Department of Civil Engineering, Helwan University, Cairo, Egypt
}

\begin{abstract}
Due to the gradual depletion in the conventional resources, searching for a more rational road construction approach aimed at reducing the dependence on imported materials while improving the quality and durability of the roads is necessary. A previous study carried out on a sample of Egyptian soil aimed at reducing the road construction cost, protect the environment and achieving sustainability. RoadCem, ground granulated blast furnace slag (GGBS), lime and ordinary Portland cement (OPC) were employed to stabilise the Egyptian clayey soil. The results revealed that the unconfined compressive strength (UCS) of the test soil increased while the free swelling percent (FSP) decreased with an increase in the total stabiliser and the curing period.

This paper discusses attempts to reach optimum stabilization through: (1) Recognizing the relationship between the UCS/FSP of stabilized soil and the stabilization parameters using artificial neural network (ANN); and (2) Performing a backward optimization on the developed (ANN) model using general algorithm (GA) to meet practical design preferences.

Keywords: clay, roadCem, slag, lime, ANN, GA, stabilisation, sustainability, artificial intelligent, swelling soil.
\end{abstract}

\section{Introduction}

Traditional road construction has proven to be very material and energy intensive. It demands import and export of enormous quantities of virgin materials. This is especially true in situations where the load bearing capacity of base soils are low or when other geotechnical conditions along the route of the road are not suitable [1]. The shortage of imported materials in many situations 
and countries leads to an increase in costs. Also higher energy costs for excavation will bring higher costs for the end user.

As a society develops, larger quantities of waste materials continue to be generated by people and these mountains of waste are also becoming an environmental problem. The possibility to use this waste material as a replacement for the imported virgin material in road construction has been recognized as an option for many years. Only a limited portion of the waste stream could be brought to use on road constructions. Due to the gradual depletion in the conventional resources, searching for a more rational road construction approach aimed at reducing the dependence on imported material while improving the quality and durability of the roads is necessary [1].

Many chemical substances have been used to stabilise soils, e. g. GGBS, lime and OPC. Mathur et al. [2] showed that properties of the soil improved when treated with lime-GGBS blends. (FSP) and linear shrinkage decreased, while the UCS and CBR values increased [2]. RoadCem is a blend of special selected substances in various percentages, it is an additive and OPC improver, used in soil stabilisation typically for road construction [1]. This results in the reduction of the required thickness of the pavement structures consequently reducing road costs and contributes to the solution of the problem of declining resource base for imported materials thus achieving sustainability [1].

A previous study was undertaken on a sample of clayey test soil chosen from east Cairo, Egypt [3]. The study concluded that the UCS increased with an increase in the total stabiliser and the curing period for the same combination. Replacement of $65 \%$ OPC by GGBS and lime increased the UCS, while replacement of $2 \%$ RoadCem by OPC caused further increase in the UCS of the test soil. The FSP of the test soil decreased with an increase in the total stabiliser and the curing period for the same combination of total stabiliser [3]. A summary of the used mixes is presented in Table 1.

Table 1: $\quad$ A summary of mix used by Ouf [3].

\begin{tabular}{|c|c|c|c|}
\hline Mix 1 & Mix 2 & Mix 3 & Mix 4 \\
\hline $2 \%$ RoadCem & $2 \%$ RoadCem & \multirow{2}{*}{$100 \%$ OPC } & $35 \%$ OPC \\
$98 \%$ OPC & $33 \%$ OPC & $50 \%$ GGBS \\
\cline { 2 - 2 } & $50 \%$ GGBS & & $15 \% \mathrm{Ca}(\mathrm{OH}) 2$ \\
\cline { 2 - 2 } & $15 \% \mathrm{Ca}(\mathrm{OH}) 2$ & \\
\hline
\end{tabular}

This paper aims to identify optimum soil stabilization for clayey soil to suit safe and economic road construction.

\section{Available data}

Ouf's findings were re-analyzed in this investigation to understand the relationship between the stabilization parameters represented by the GGBS\%, Lime $\%$, OPC $\%$, RoadCem and curing period versus both the UCS and the FSP, using artificial neural networks. A genetic optimization was applied in a 
backward technique to reach an optimal set of stabilization parameters under practical site considerations.

Table 2 shows a summary of the information extracted from the tests presented in [3]. It can be noticed from the table that the maximum and minimum UCS after stabilization were $3682 \mathrm{kN} / \mathrm{m}^{2}$ and $687 \mathrm{kN} / \mathrm{m}^{2}$ respectively while the maximum and minimum FSP were $30 \%$ and $0 \%$ respectively, which have a lot of variation based on stabilizing parameters.

\section{Research methodology}

The methodology had two stages. Initially Neural Tools 5.5 was used and then the UCS was predicted for both training and testing. Due to the neural Tools 5.5 has no transparent link between inputs and outputs, it is very difficult to perform backward analysis to determine the optimum set of inputs that may result in certain output. Accordingly, a more transparent ANN model for neural network was developed. A backward optimization as such is proposed on the same ANN model after training and testing. The variables in the proposed optimization problem are the stabilization parameters with an objective to reach a certain UCS keeping the FSP within a certain range. To implement the proposed approach, an Excel application was developed. After training and testing, a friendly-user interface to optimize the UCS for any set of stabilization parameters was used. The user can set any stabilization parameters which are linked automatically with the two ANNs sheets to reflect the expected UCS and FSP. With the automatic link between the stabilization parameters, this interface can function as an optimization model. Upon activating EVOLVER (GAs based optimization solver for Excel) can be used so that the user can get the optimum set of stabilization parameters.

\subsection{Behavior prediction}

The first stage is to identify the behavior of soil in terms of UCS and FSP under any combination of stabilization parameters. In this research ANN was proposed to be used for this prediction. First, an investigation for the suitability of using ANN in predication was carried out, and then a spreadsheet ANN model was developed which suit the current study.

To start the investigation, a simple to use ANN add-ins (Neural Tools 5.5) for Excel was used and the UCS was predicted. The results showed that, ANN can be used in predicting the UCS of the stabilized soil with good accuracy. A detailed error analysis was performed for the training and testing cases and these are shown in figures 3 and 4 respectively. While figures 5 and 6 show the error distribution for the FSP training and testing cases respectively.

It is very difficult to perform backward analysis to determine the optimum set of inputs that may result in certain output. Accordingly, a more transparent model for neural network was developed. 


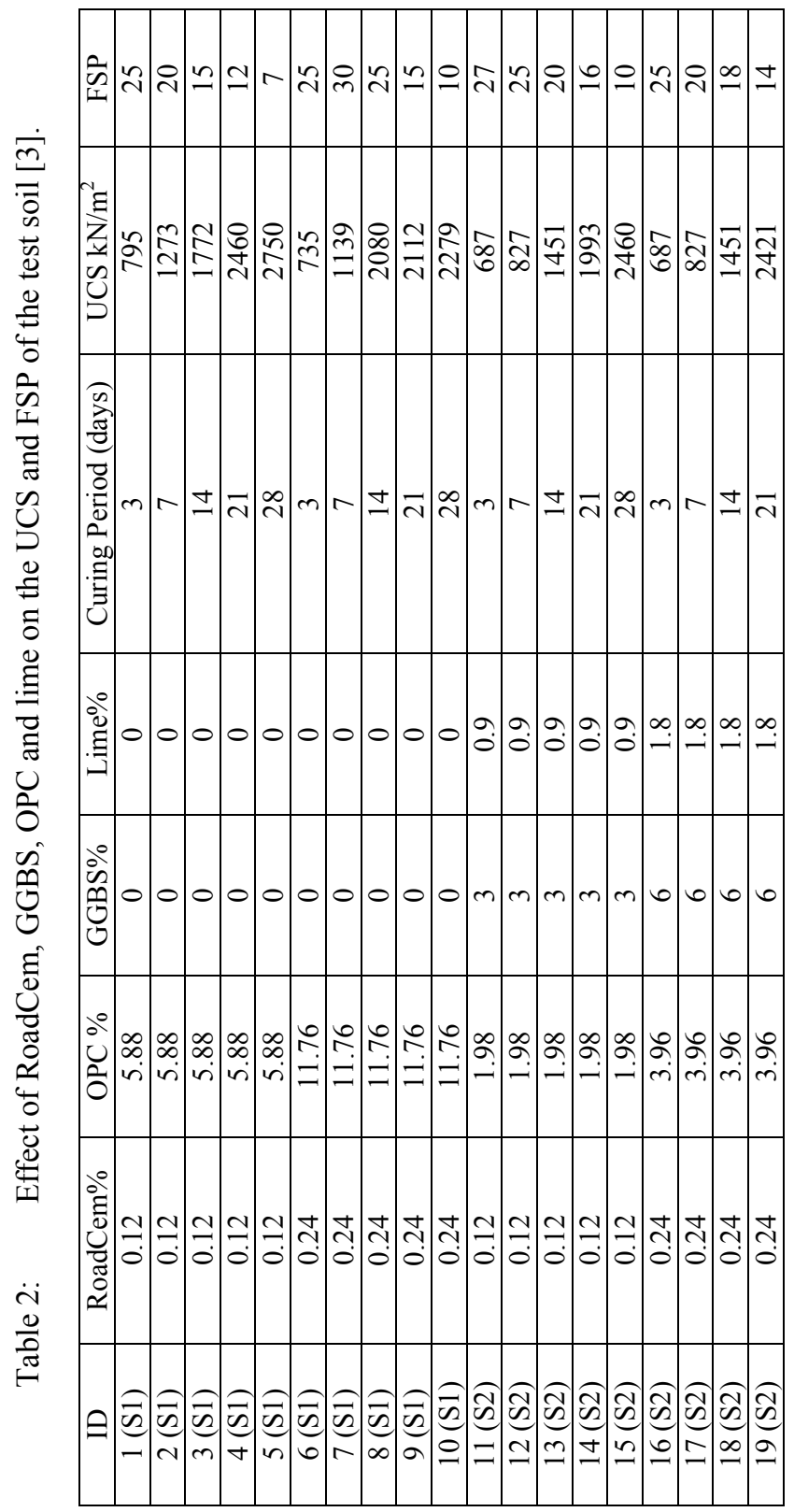




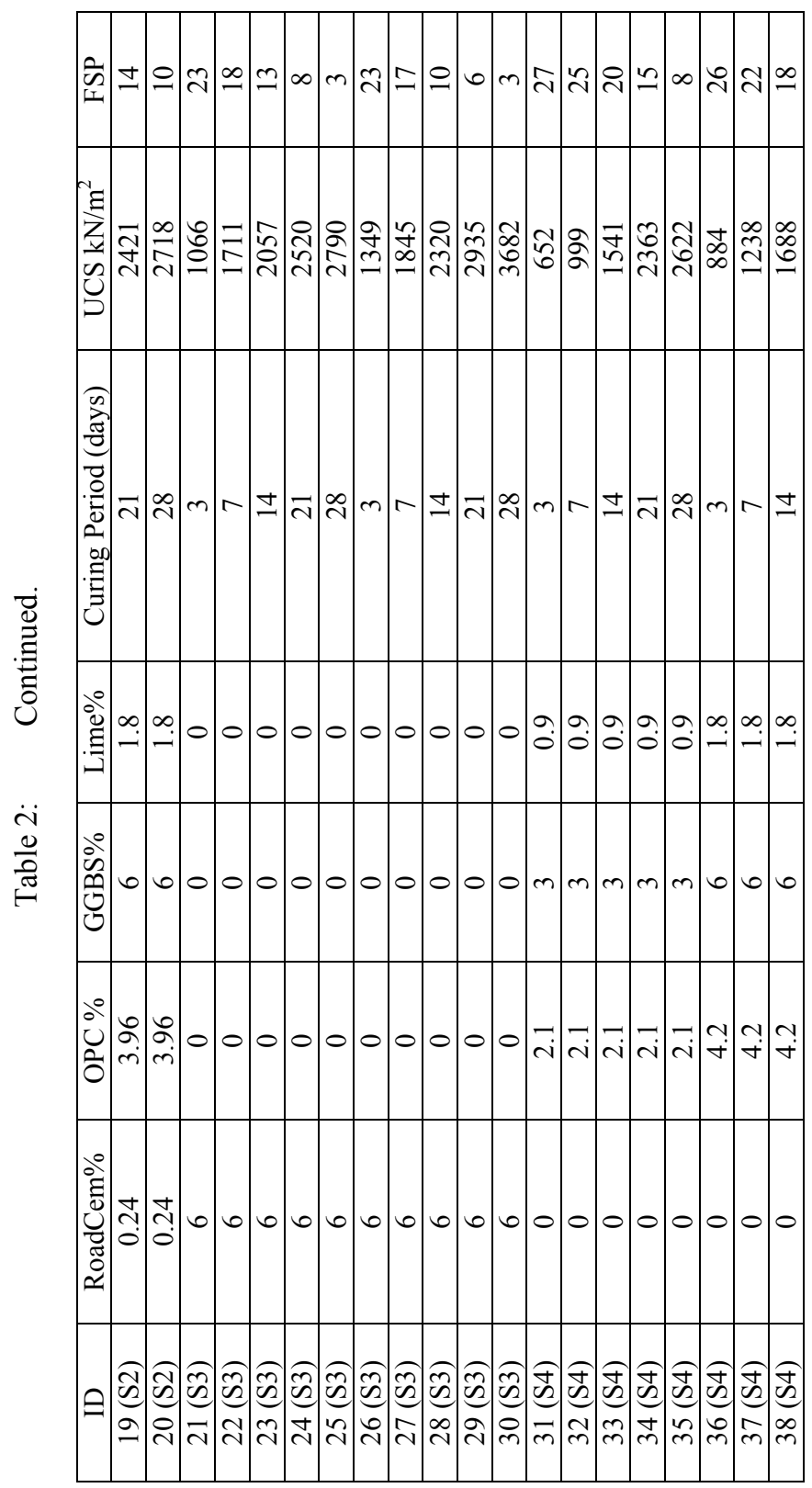




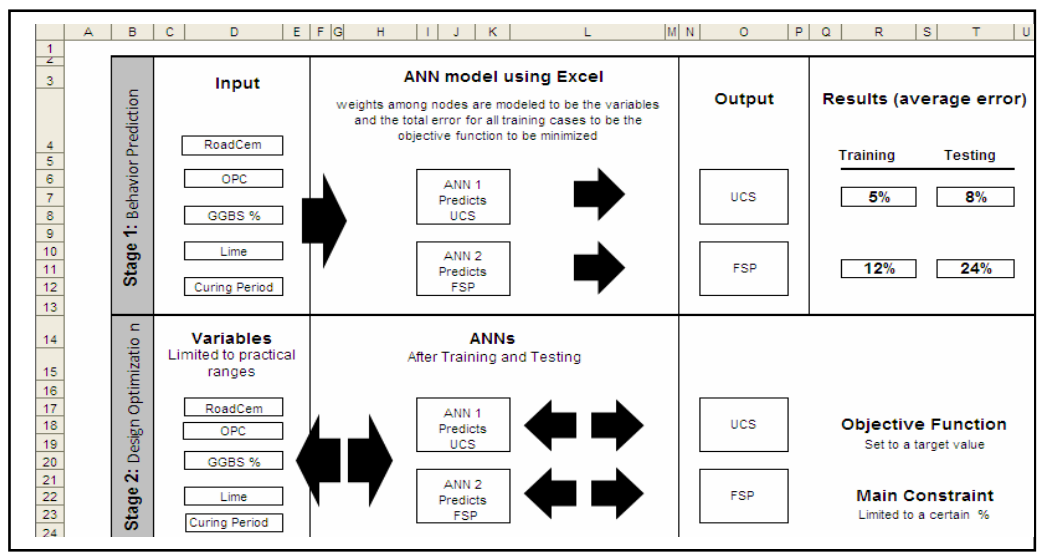

Figure 1: Research methodology for optimum soil stabilization.

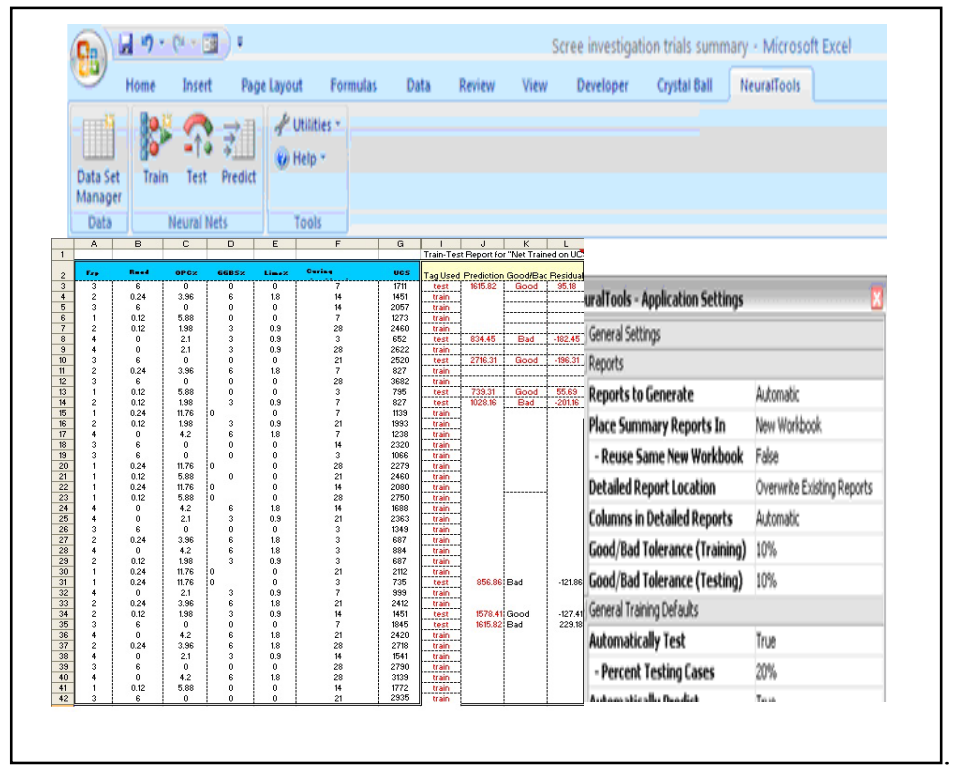

Figure 2: $\quad$ UCS prediction using ANN (NeuroTool5.5 screenshot)

The developed model imitates the architecture and the process flow of an ANN with one hidden layer using spreadsheet modelling similar to Hegazy and Ayed [4]. The learning process was carried out through optimization where the errors between ANN predictions and actual results for the training set were minimized. The variables are the weights between ANN different layers. As such, the core of the model is an optimization formulation using a spreadsheet. Once the weights were determined, the model can then be tested using other set 


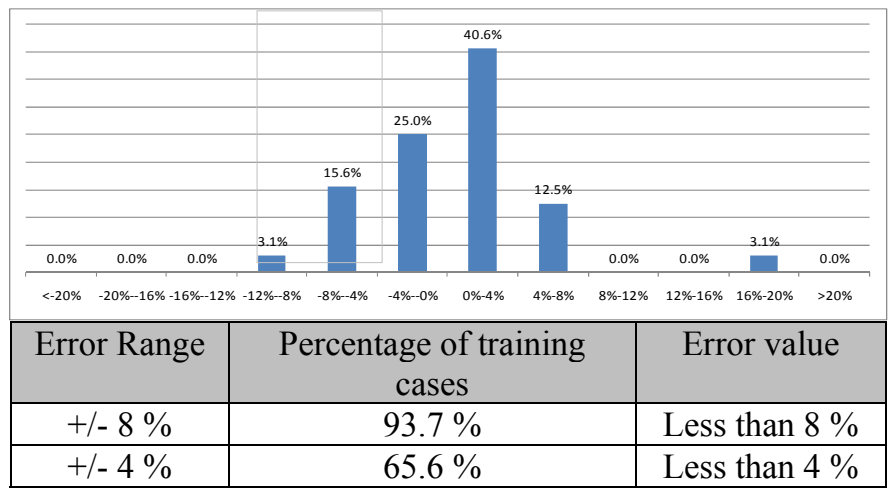

Figure 3: $\quad$ Error distributions for the UCS training.

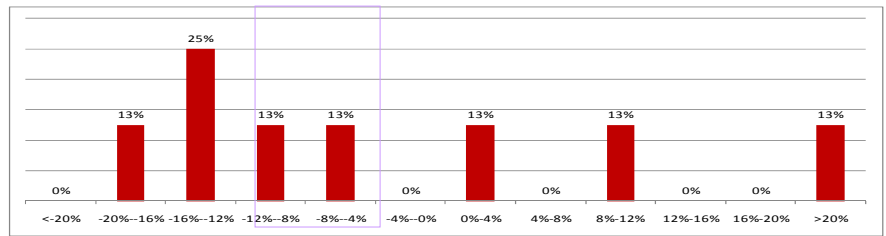

\begin{tabular}{|c|c|c|}
\hline $\begin{array}{c}\text { Error } \\
\text { Range }\end{array}$ & $\begin{array}{c}\text { Percentage of testing } \\
\text { cases }\end{array}$ & Error value \\
\hline$+/-16 \%$ & $77.00 \%$ & Less than $16 \%$ \\
\hline$+/-12 \%$ & $52.00 \%$ & Less than $12 \%$ \\
\hline$+/-8 \%$ & $26.00 \%$ & Less than $8 \%$ \\
\hline$+/-4 \%$ & $13.00 \%$ & Less than $4 \%$ \\
\hline
\end{tabular}

Figure 4: $\quad$ Error distributions for the UCS testing.

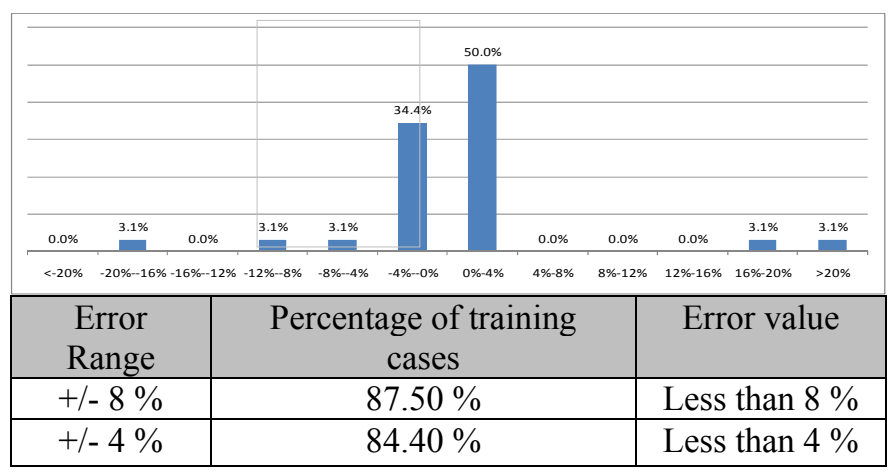

Figure 5: $\quad$ Error distributions for the FSP training. 


\begin{tabular}{|c|c|c|c|}
\hline & & & \\
\hline & & & \\
\hline & & & \\
\hline & & & \\
\hline
\end{tabular}

Figure 6: $\quad$ Error distributions for the UCS testing.

of data then used to predict the outputs for any set of inputs. The reader can refer to [4] for more details. To train the ANN, 32 randomly selected cases were considered while the 8 rest cases were used in the testing phase.

\subsection{Design optimization}

As the Excel based ANN model of stage 1 with their hidden nodes link the stabilization parameters in the input layer with the design criteria in the output layer, the model can function as an optimization model in a reverse order in which the UCS (predicted) represents the objective function and the stabilization parameters (in the input layer) represent the decision variables to be determined (Stage 2).

A backward optimization as such is proposed on the same ANN model after training and testing. The variables in the proposed optimization problem are the stabilization parameters that can be varied to be determined under practical site and design constraints with an objective to reach a certain UCS keeping FSP within a certain range.

\section{Implementation and testing}

To implement the proposed approach, an Excel application was developed in three sheets. The first and the second are the ANNs models for the USC and FSP respectively. After training and testing, these two sheets represent the UCS and FSP predictors. The third sheet however represents the friendly-user interface to optimize the UCS for any set of stabilization parameters. The user can set any stabilization parameters preferences which are linked automatically with the two ANNs sheets to reflect the expected UCS and FSP, this interface can function as an optimization model. Upon activating EVOLVER, (GAs based optimization 
solver for Excel) can be used so that the user can get the optimum set of stabilization parameters.

To test the developed model, practical ranges for the UCS were obtained from [5] who proposed guiding values between $345 \mathrm{KN} / \mathrm{m}^{2}$ and $1700 \mathrm{KN} / \mathrm{m}^{2}$ for base construction in low cost roads. The design parameters should be decided both on cost and performance, including all parameters involved to achieve an acceptable UCS value. For example, the minimum and maximum UCS is set to be 1000 $\mathrm{KN} / \mathrm{m}^{2}$ and $1800 \mathrm{KN} / \mathrm{m}^{2}$, respectively. Evolver is then used to maximize the UCS within the given range of minimum and maximum. All other stabilization parameters in addition to the FSP are also constrained within their specified ranges.

Optimization results show that it is possible to reach a UCS of $1791 \mathrm{KN} / \mathrm{m}^{2}$ with a $9 \%$ FSP by considering a $0.12 \%$ of RoadCem, $6.2 \%$ of OPC, $0 \%$ of GGBS and lime at $30^{\circ} \mathrm{C}$ curing temperature for 19 days. Ouf and Al-Hakim [6] also found using the same model, that it is possible to reach a UCS of $1792 \mathrm{kN} / \mathrm{m}^{2}$ with a 5\% FSP by considering an $11 \%$ Lime/GGBS and a 7\% binder (Lime + GGBS) at $37^{\circ} \mathrm{C}$ curing temperature for 27 days when using a test soil similar to some extent to the soil used in this investigation.

\section{Environmental effects}

Environmental conditions generally affect the performance of road pavements. For example, high temperature can accelerate hydration of stabilisers in road materials, resulting in loss in flexibility and consequent ravelling of the aggregate and brittle fracture of the layer.

High rainfall can result in a change in the moisture content of the subgrade materials. Under poor drainage conditions, materials adversely affect the pavement structure and its performance under traffic. However, when RoadCem is used this mode of failure is largely eliminated [1]. It is noted that when RoadCem is used the mechanism of interaction between pavement structure and carbon dioxide in the atmosphere is totally different than in the case of traditional materials and as a result carbonation is not a problem [1]. The pavement structure may actually be isolated from carbon dioxide which leads to elimination of carbonation.

\section{Conclusions}

The main conclusions can be summarized as:

1. A simple-to-use Neural Tools 5.5 can be used in predicting the UCS of the stabilized soil for both training and testing.

2. A more transparent model for neural network was developed and a novel approach to understand the relationship between the stabilization parameters versus both the UCS and the FSP using ANNs that expedite the way for optimum soil stabilization was presented.

3. This paper reached the optimum stabilization for clay soil by: (1) Recognizing the relationship between the UCS of stabilized soil and the stabilization 
parameters. Two ANNs were developed to predict the UCS and the FSP based on any set of stabilization parameters. The behaviors were successfully investigated using the ANN approach; the acceptable errors for both training and testing confirm this conclusion; and (2) Performing a backward optimization using GA which shows its ability to determine an optimum set of stabilization parameters under practical constrains.

\section{Acknowledgement}

The author would like to thank Dr. Ahmed El-hakim, Post Doctorate Fellow, AUC/KAUST, The American University in Cairo, Egypt, for his cooperation in development of the neural network model.

\section{References}

[1] Marjanovic, P., Egyed, C.E.G., De La Roij, P and de La Roij, R., "The Road to The Future, The Economic Immobilizer with more security for mankind: Manual working with ImmoCem", PowerCem Technologies, (2008).

[2] Mathur, S, Prasad, P S and Kamaraj, C., "Feasibility of Copper Slag-Fly Ash-Soil Mix as a Road Construction Material", Transportation Research Board, Low-Volume Roads 1989: 2 (2007).

[3] Ouf, M.S., "Assesment of Using RoadCem As An Effective Soil Stabiliser For The Egyptian Soil", IC-NOCMAT, International Conference on NonConventional Materials and Technologies: Ecological Materials and Technologies for Sustainable Building National Center for Housing and Building, Cairo, Egypt (2010).

[4] Hegazy T. and Ayed, A.,"A Neural Network Model for Parametric Cost Estimation of Highway Projects," Journal of Construction Engineering and Management, ASCE, Vol. 24, No. 3, (1998).

[5] Odier, L, Millard, R.S, Dos Santos, P and Mehta, S.R., "Low Cost Roads", Butterworths (London), (1971).

[6] Ouf, M.S and Al-Hakeem, A.A., "Optimum Soil Stabilization using A Mix of Artificial Intelligence Techniques", International Conference on Computational in Engineering, (Code 2009), Seoul, South Korea, (2009). 